\title{
Ge/SiGe Quantum Well p-i-n Structures for Uncooled Infrared Bolometers
}

\author{
Fatih Bilge Atar, Alper Yesilyurt, Mehmet Cengiz Onbasli, Oguz Hanoglu, and Ali K. Okyay
}

\begin{abstract}
The temperature dependence of current is investigated experimentally for silicon-germanium (Si-Ge) multi-quantum-well p-i-n devices on $\mathrm{Si}$ substrates as uncooled bolometer active layers. Temperature coefficient of resistance values as high as $-5.8 \% / \mathrm{K}$ are recorded. This value is considerably higher than that of even commercial bolometer materials in addition to being well above the previous efforts based on CMOS compatible materials.
\end{abstract}

Index Terms-Bolometers, germanium, quantum wells, silicon.

\section{INTRODUCTION}

$\mathbf{T}$ HE commercialization of the uncooled microbolometer infrared (IR) detector technology has ignited an extensive market growth of thermal IR cameras for use in automobile night vision, environmental monitoring, and security applications. There has been a growing interest in decreasing the cost and increasing the efficiency of uncooled microbolometer detectors [1]. Unlike photon detectors, microbolometers do not require bulky and expensive cryogenic cooling, enabling compact and lightweight designs [1]. The active layer of a microbolometer is a temperature-dependent resistor. Change in temperature due to IR radiation is usually measured as a resistance change of the active layer and is translated into an electrical signal by read out integrated circuit (ROIC).

The active material used as thermistor of a microbolometer should have high temperature sensitivity, have low noise, and be compatible with standard Si CMOS processes for lowcost manufacturing and integration with ROIC. Temperature coefficient of resistance (TCR) is defined as the percent change of a material's electrical resistance $R$ with unit temperature difference

$$
T C R=\frac{1}{R} \frac{\partial R}{\partial T}
$$

Manuscript received July 29, 2011; accepted August 2, 2011. Date of publication September 18, 2011; date of current version October 26, 2011. This work was supported by EU FP7 Marie Curie IRG Grant 239444, TUBITAK EEEAG Grants 108E163 and 109E044, Turkish Ministry of Industry and Trade Seed Fund and TUBITAK BIDEP. The review of this letter was arranged by Editor C. Jagadish.

F. B. Atar, A. Yesilyurt, O. Hanoglu, and A. K. Okyay are with the Department of Electrical and Electronics Engineering and Institute of Materials Science and Nanotechnology, Bilkent University, 06800 Ankara, Turkey (e-mail: fbatar@ee.bilkent.edu.tr; aokyay@ee.bilkent.edu.tr).

M. C. Onbasli is with the Department of Materials Science and Engineering, Massachusetts Institute of Technology, Cambridge, MA 02139 USA.

Color versions of one or more of the figures in this letter are available online at http://ieeexplore.ieee.org.

Digital Object Identifier 10.1109/LED.2011.2164390
Microbolometers based on n-type single-crystalline $\mathrm{Si}$ active layers monolithically integrated with ROIC are demonstrated, but TCR values are low $(-0.65 \% / \mathrm{K})$ [2]. Vanadium oxide $\left(\mathrm{VO}_{x}, T C R \approx-2.0 \% / \mathrm{K}\right)$ [3], yttrium barium copper oxide (YBCO, TCR $\approx-3.5 \% / \mathrm{K}$ ) [4], and amorphous Si $(\alpha-\mathrm{Si}, T C R \approx-2.5 \% / \mathrm{K})$ [5] are used as active materials in microbolometers. $\mathrm{VO}_{x}$ and $\mathrm{YBCO}$ are not compatible with standard Si CMOS processes, therefore resulting in high manufacturing and integration costs. $\alpha$-Si-based sensors suffer from high $1 / f$ noise [1].

Polycrystalline SiGe (poly-SiGe, TCR $=2 \% / \mathrm{K}$ ) [6], $\alpha$ SiGe $(T C R=4.7 \% / \mathrm{K})$ [7], and SiGe multi-quantum-well (MQW) $(T C R<3.5 \% / \mathrm{K})$ [8]-[12] structures have gathered much attention due to their high TCR values. Among these, single crystal structures of SiGe MQWs exhibit the best $1 / f$ noise performance due to high level of order and material quality. Bolometer devices with SiGe MQW active layers can be manufactured using low-temperature hybridization technique [11]. Recently, by incorporating carbon in SiGe quantum well layers, TCR values up to $4.5 \% / \mathrm{K}$ were also demonstrated [13]. High Ge content in such MQW structures are shown to increase TCR values, but all efforts so far were limited to below $35 \% \mathrm{Ge}$ content $(T C R<3.5 \% / \mathrm{K})$ due to the large lattice mismatch between $\mathrm{Si}$ and $\mathrm{Ge}$, limiting the TCR value to below $3.5 \% / \mathrm{K}$ in SiGe layers without incorporating other species [8]-[12]. Therefore, there is a need for further increasing the TCR of CMOS compatible materials with good crystal quality, beyond the improvement levels obtained using high Ge content SiGe MQW structures. This, however, requires innovative designs. In this letter, to address this need, we demonstrate Ge/SiGe MQW structures embedded in a p-i-n diode architecture.

\section{EXPERIMENT}

\section{A. Device Fabrication}

The design used in our experiments is shown in Fig. 1(a). A recently developed epitaxial technique is used to grow the designed stack [14], [15]. Si p-type (100) wafers $(10-20 \Omega \cdot \mathrm{cm})$ are used as starting substrates. Since epitaxial growth is very sensitive to surface preparation and contamination, standard RCA-1 (organic removal) and RCA-2 (metal removal) cleaning procedures were performed prior to epitaxial growth. The wafers were immediately loaded into an Applied Materials Centurion reduced pressure chemical vapor deposition system. The wafers were baked for $5 \mathrm{~min}$ at $1000{ }^{\circ} \mathrm{C}$ in $\mathrm{H}_{2}$. This step ensures the removal of any native oxide from the surface. A 180-nm-thick boron-doped Ge layer is grown at $400{ }^{\circ} \mathrm{C}$, and the wafers are annealed for $20 \mathrm{~min}$ at $825{ }^{\circ} \mathrm{C}$ in $\mathrm{H}_{2}$. This 


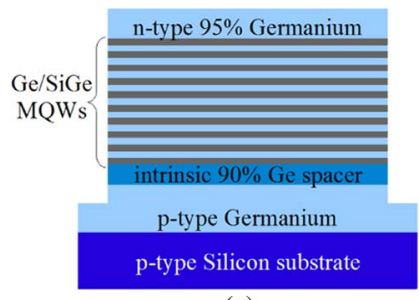

(a)

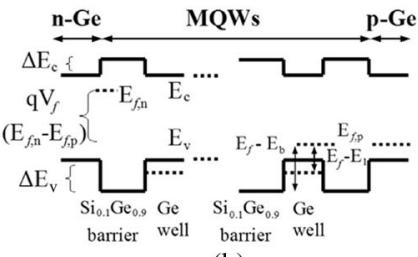

(b)
Fig. 1. (a) MQW layers embedded in a p-i-n architecture. (b) Theoretical energy band diagram.

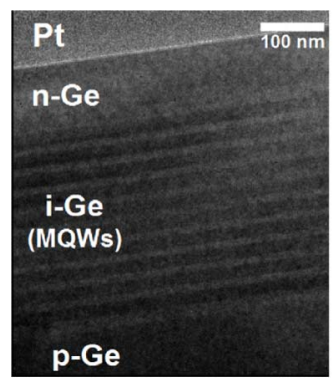

(a)

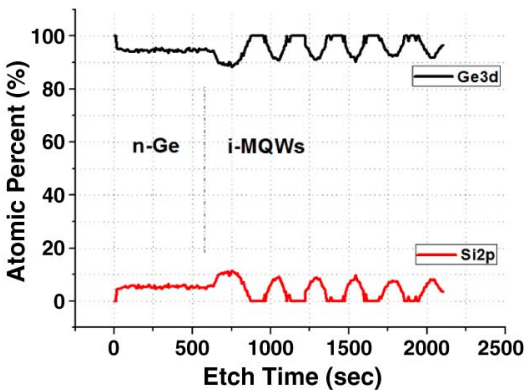

(b)
Fig. 2. (a) TEM image of the MQW regions. (b) X-ray photoelectron spectroscopy results.

step provides smooth surface by enhanced surface diffusion and low defect density by dislocation annihilation [14], [15], resulting in a virtual Ge substrate. Undoped MQW regions are grown on such virtual Ge substrates. Alternating Ge well regions and $\mathrm{SiGe}$ barrier regions are grown at $400{ }^{\circ} \mathrm{C}$ without any intermediate annealing step to avoid the interdiffusion of $\mathrm{Si}$ and Ge and the diffusion of doping species from highly doped regions. A 100-nm-thick n-type Ge-rich layer is grown as the top layer. Fig. 1(b) shows the energy band diagram of the grown p-i-n structure.

Fig. 2(a) shows a cross-sectional transmission electron microscope (TEM) image of the grown structure with alternating quantum well barrier (ten pairs) of 10-nm Ge wells and 20-nm $\mathrm{Si}_{0.1} \mathrm{Ge}_{0.9}$ barriers. X-ray photoelectron spectroscopy combined with in situ etching is used to determine layer compositions as a function of distance from the surface as shown in Fig. 2(b). The quantum well regions are $100 \% \mathrm{Ge}$, and the barriers are composed of $10 \% \mathrm{Si}$ and $90 \% \mathrm{Ge}$.

Vertical mesa structures are patterned by optical lithography followed by dry etching. The unsatisfied bonds at the surface of the defined mesa structures may cause an increased generationrecombination rate resulting in a high surface leakage current. A 100 -nm-thick $\mathrm{Si}_{3} \mathrm{~N}_{4}$ is used to electrically passivate the sidewalls of the mesa structure to suppress the surface leakage current. Aluminum electrodes are used for electrical contacts to doped $\mathrm{p}$ - and n-regions.

\section{B. Results and Discussion}

In earlier reports, p-type $\mathrm{Si}_{1-x} \mathrm{Ge}_{x}$ quantum wells and p-type $\mathrm{Si}$ quantum barriers [8] and intrinsic SiGe/Si quantum wells embedded in p-i-p structures [10] were demonstrated. It was shown that TCR increased with the energy difference between the Fermi level and the barrier energy $\left(E_{f}-E_{b}\right)$ [Fig. 1(b)]. This energy difference was increased with higher Ge content in the quantum wells yielding higher barrier energies $E_{b}$ 's and increased TCR values. All efforts so far have been limited to below $35 \%$ Ge content $(T C R<3.5 \% / K)$ due to $4.2 \%$ lattice mismatch between $\mathrm{Si}$ and Ge resulting in the formation of excessive defects and dislocations [8]-[12]. TCR values of such layers can be further increased if quantum wells are located in the intrinsic region of a p-i-n structure. Thermionic emission current dominates over sequential resonant tunneling and phonon-assisted tunneling current components in an MQW $\mathrm{p}-\mathrm{i}-\mathrm{n}$ device, at room temperature [16]. The thermionic current in a p-i-n quantum well structure can be expressed as [17]

$$
I_{\mathrm{th}} \propto \frac{\sqrt{2 \pi m(k T)^{3}}}{\pi \hbar^{2}} \exp \left(-\frac{q V_{f}-E_{1}-E_{f}}{k T}\right)
$$

where $V_{f}$ is the applied forward bias, $E_{f}$ is the relative position of the Fermi level, and $E_{1}$ is the first quantized energy state in the valence band quantum well. At room temperature, the exponential term is responsible from strong temperature dependence of the current. In a p-i-n configuration, the relative position of the Fermi level in the intrinsic quantum wells can be considerably lower than the ground state energy, and when combined with the $q V_{f}$ term, resulting in high dependency of the current to the temperature. Taking the derivative of current $I_{\text {th }}$ with respect to temperature $T$, as in [8], we also expect that TCR should increase with increasing forward bias $V_{f}$.

To measure the TCR values, electrical contacts to the devices are obtained by gold bonding wires, and the samples are placed on Torrey Pines Scientific's Model IC25 heating/chilling plate. Samples were covered by a custom enclosure to provide better thermal isolation. Measurements at each temperature point are carried out after waiting for thermal stabilization. A calibrated temperature sensor, AD590LF, is attached next to the sample surface to monitor the surface temperature, and it is recorded simultaneously with the mesa current.

$I-V$ characteristics of the MQW p-i-n devices obtained at $15{ }^{\circ} \mathrm{C}, 20{ }^{\circ} \mathrm{C}, 25{ }^{\circ} \mathrm{C}$, and $30{ }^{\circ} \mathrm{C}$ are plotted in Fig. 3. Temperature-dependent resistance values are extracted from the measured $I-V$ values at different voltage bias points, and TCR values are obtained by calculating the best fit to resistanceversus-temperature relation (inset in Fig. 3). In accordance with the theory, as the applied voltage to the p-i-n device is swept from reverse bias to forward bias, TCR increases with increasing voltage until around $0.2-\mathrm{V}$ forward bias. Fig. 4 plots the obtained TCR values for bias voltage between -0.25 and $0.25 \mathrm{~V}$, exhibiting TCR values up to $-5.8 \% / \mathrm{K}$. For bias voltages higher than $0.2 \mathrm{~V}$, TCR decreases sharply, which is believed to be due to high field effects, but this requires further investigation.

Noise performance of Ge/SiGe MQW devices is characterized by measuring the noise spectral density between 0.1 and $100 \mathrm{~Hz}$, under different biasing conditions. Noise characterization setup and measured results are shown in Fig. 5. At a frequency of $10 \mathrm{~Hz}$, the total noise is measured to be around $800 \mathrm{nV} / \sqrt{ } \mathrm{Hz}$ for bias voltages less than $0.2 \mathrm{~V}$. Although no effort was made to optimize the noise characteristics, this 


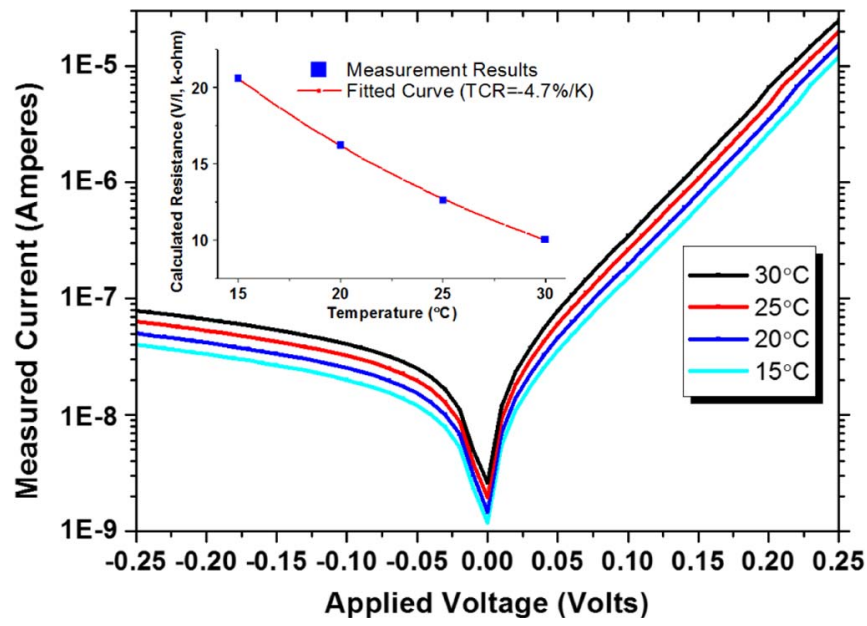

Fig. 3. Current-voltage characteristics at different temperatures. The inset shows the temperature-dependent change of the calculated resistance values at $0.25-\mathrm{V}$ bias voltage.

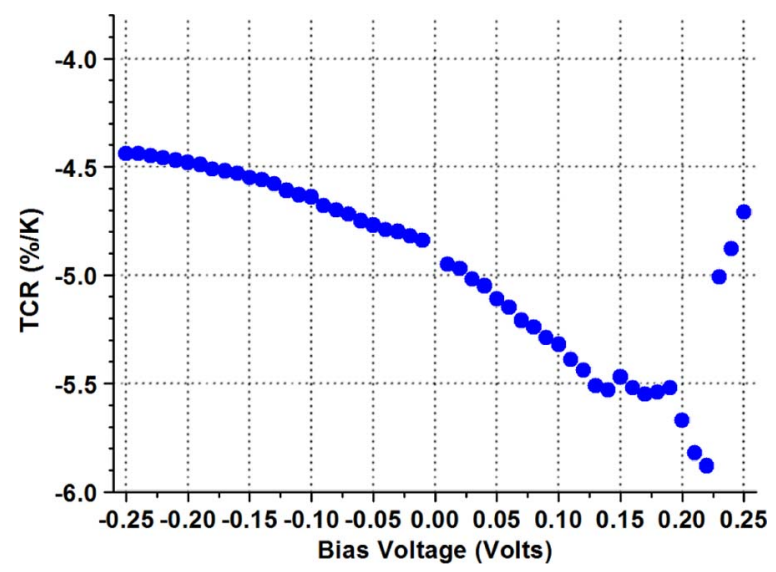

Fig. 4. Measured TCR values of the devices at different bias voltages.

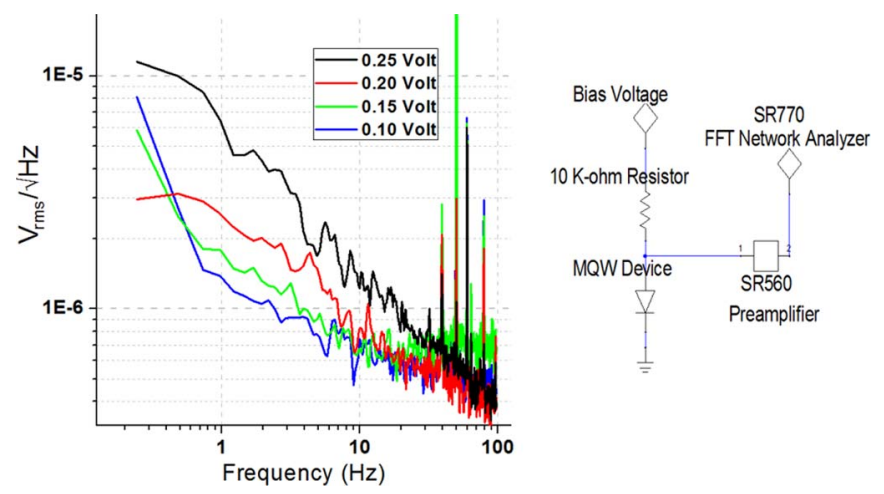

Fig. 5. Measured noise spectral density at different bias voltages and the noise measurement setup.

is similar to the noise performance reported earlier [8]-[12]. Large peaks are due to $50-\mathrm{Hz}$ mains and harmonics.

\section{CONCLUSION}

The use of SiGe p-i-n MQW structures as a high TCR device has been demonstrated. A novel CMOS compatible heteroepitaxy technique, i.e., MHAH, is used for the layer growth. MHAH enables the fabrication of high Ge content and low-defect-density MQW structures on Si substrates. Experimental TCR values as high as $-5.8 \% / \mathrm{K}$ are recorded from such structures. This is considerably higher than prior reports based on CMOS compatible materials, current commercial high TCR materials, and SiGe MQW structures.

\section{REFERENCES}

[1] A. Rogalski, "Review infrared detectors: Status and trends," Prog. Quantum Electron., vol. 27, no. 2/3, pp. 59-210, 2003.

[2] S. Eminoglu, D. S. Tezcan, M. Y. Tanrikulu, and T. Akin, "Low-cost uncooled infrared detectors in CMOS process," Sens. Actuators A, Phys., vol. 109, no. 1/2, pp. 102-113, Dec. 2003.

[3] C. Chen, X. Yi, J. Zhang, and X. Zhao, "Linear uncooled microbolometer array based on $\mathrm{VO}_{x}$ thin films," Infrared Phys. Technol., vol. 42, no. 2, pp. 87-90, Apr. 2001.

[4] M. Almasri, D. P. Butler, and Z. Çelik-Butler, "Self supporting uncooled infrared microbolometers with low thermal mass," J. Microelectromech. Syst., vol. 10, no. 3, pp. 469-476, Sep. 2001

[5] E. Mottin, J. Martin, J. Ouvrier-Buffet, M. Vilain, A. Bain, J. Yon, J. L. Tissot, and J. P. Chatard, "Enhanced amorphous silicon technology for $320 \times 240$ microbolometer arrays with a pitch of $35 \mu \mathrm{m}$," Proc. SPIE, vol. 4369, pp. 250-256, Oct. 2001

[6] S. Sedky, P. Fiorini, M. Caymax, C. Baert, L. Hermans, and R. Mertens, "Characterization of bolometers based on polycrystalline silicon germanium alloys," IEEE Electron Device Lett., vol. 19, no. 10, pp. 376-378, Oct. 1998.

[7] M. Moreno, R. Ambrosio, A. Torres, A. Kosarev, M. Garcia, and J. Mireles, "Measurements of thermal characteristics in silicon germanium un-cooled micro-bolometers," Phys. Stat. Sol. (C), vol. 7, no. 3/4, pp. $1172-1175$, Apr. 2010

[8] S. G. E. Wissmar, H. H. Radamsson, Y. Yamamoto, B. Tillack, C. Vieider, and J. Y. Andersson, "SiGe quantum well thermistor materials," Thin Solid Films, vol. 517, no. 1, pp. 337-339, Nov. 2008.

[9] L. Di Benedetto, M. Kolahdouz, B. G. Malm, M. Östling, and H. H. Radamson, "Strain balance approach for optimized signal-to-noise ratio in SiGe quantum well bolometers," in Proc. Eur. Solid State Device Res. Conf., Sep. 14-18, 2009, pp. 101-104.

[10] M. Kolahdouz, A. Afshar Farniya, M. Östling, and H. H. Radamson, "The performance improvement evaluation for SiGe-based IR detectors," Solid State Electron., vol. 62, no. 1, pp. 72-76, Aug. 2011.

[11] J. Y. Andersson, P. Ericsson, H. H. Radamson, S. G. E. Wissmar, and M. Kolahdouz, "SiGe/Si quantum structures as a thermistor material for low cost IR microbolometer focal plane arrays," Solid State Electron., vol. 60, no. 1, pp. 100-104, Jun. 2011.

[12] F. Forsberg, N. Roxhed, P. Ericsson, S. Wissmar, F. Niklaus, and G. Stemme, "High-performance quantum-well silicon-germanium bolometers using IC-compatible integration for low-cost infrared imagers," in Proc. IEEE Int. Conf. Solid-State Sens., Actuators, Microsyst., Denver, CO, Jun. 21-25, 2009, pp. 2214-2217.

[13] H. H. Radamson, M. Kolahdouz, S. Shayestehaminzadeh, A. Afshar Farniya, and S. Wissmar, "Carbon-doped single-crystalline SiGe/Si thermistor with high temperature coefficient of resistance and low noise level," Appl. Phys. Lett., vol. 97, no. 22, pp. 223 507-1-223 507-2, Nov. 2010.

[14] A. Nayfeh, C. O. Chui, K. C. Saraswat, and T. Yonehara, "Effects of hydrogen annealing on heteroepitaxial-Ge layers on Si: Surface roughness and electrical quality," Appl. Phys. Lett., vol. 85, no. 14, pp. 2815-2817, Oct. 2004.

[15] A. K. Okyay, A. M. Nayfeh, K. C. Saraswat, T. Yonehara, A. Marshall, and P. C. McIntyre, "High-efficiency metal-semiconductor-metal photodetectors on heteroepitaxially grown $\mathrm{Ge}$ on Si," Opt. Lett., vol. 31, no. 17, pp. 2565-2567, Sep. 2006.

[16] B. F. Levine, "Quantum well infrared photodetectors," J. Appl. Phys., vol. 74, no. 8, pp. R1-R81, Oct. 1993.

[17] E. Aperathitis, A. C. Varonides, C. G. Scott, D. Sand, V. Foukaraki, M. Androulidaki, Z. Hatzopoulos, and P. Panayotatos, "Temperature dependence of photocurrent components on enhanced performance GaAs/AlGaAs multiple quantum well solar cells," Sol. Energy Mater. Sol. Cells, vol. 70, no. 1, pp. 49-69, Dec. 2001. 